\title{
Experimental Study of the Compressive Performance of Life Jacket Use Polyurethane Foam for Blast Wave Protection
}

\author{
Li Maohui ${ }^{1,2, a}$, Bai Chunhua ${ }^{1, b}$, Shi Meiwu ${ }^{2, c}$, Wei Yanpeng ${ }^{3}$, Zhou Hong ${ }^{2}$ \\ and Shen Yuhong ${ }^{2}$
}

${ }^{1}$ State Key Laboratory of Explosion Science and Technology, Beijing Institute of Technology, Beijing 100081, China

${ }^{2}$ The Quartermaster Equipment Research Institute of the General Logistics Department of the PLA, Beijing 100010, China

${ }^{3}$ Institute of Mechanics, Chinese Academy of Sciences, Beijing 100080, China

alimaohui62023@sina.com, bchunhuabai@bit.edu.cn,

${ }^{*}$ Corresponding author Tel. :+(86)01066727145.

E-mail address: limaohui62023@sina.comcshimeiwu@263.net

Key words: underwater blast injury, polyurethane foam, compressive performance

\begin{abstract}
Underwater blast injury is a common war injury during combat around islands or fighting for beachhead, and is also ordinary in underwater engineering construction blast. Adding an extra underwater blast wave protection layer and Integration with life jacket is a reasonable protection measure. Four different low mass density closed cell polyurethane foams used in life jacket have been tested by quasi-static material testing machine, and the dynamic mechanical behavior at strain rates of $10^{-3} \sim 10^{-1} \mathrm{~s}^{-1}$ has been analyzed. The yield strength has been tested with Split Hopkinson Pressure Bar. Experimental results show that the compressive performance of the polyurethane foam is sensitive to the strain rates. The polyurethane foams' yield strength shows rising trend along with strain rates increase. Under the condition of the plateau yield strength below human's allowable pressure, the energy absorption performance of the polyurethane foams has been analyzed and in contrast.
\end{abstract}

\section{Introduction}

Underwater blast injury is a common wound in a sea battle, and is also ordinary in underwater engineering construction blast. The injury is caused by the pressure wave which produced by all kinds of explosive weapons when blast in water. After the medium-term of last century, scholars ${ }^{[1-4]}$ of domestic and foreign have done massive research efforts on the physics, mechanism and medical care of the injury, and have acquired a lot of achievement. However, there is little research work done on underwater blast injury protection, especially about human body protection materials and manufactures. No report correlate has been found until now. For practical purpose, it is necessary to combine $^{1}$ the underwater blast injury protection with life jacket, and it is also very feasible. At present, there have been no discussions about the life jacket floating materials which not only having good life-saving properties but also good blast wave energy absorption capacity.

The purpose of common life jacket is life saving for human body in water. The floating body foam of life jacket is elastic body material, and the primary property is stable buoyant force offering. Generally, the energy absorbing capacity of the elastic body foam is not high when the compressive stress approaching to human body tolerance limit. As a result, underwater blast wave protection requires the floating body foam having much better energy absorbing capacity. When encountered explosion wave, the protection materials should absorb the impact energies as much as possible, and attenuate some extent impacting energy effectively, avoiding injuries happen.

The experiment is based on the special energy absorption and cushioning properties of the porous materials. The testing materials are four low density closed cell polyurethane foams and one typical active military life jacket floating material NBR/PVC foam. Through quasi-static and higher strain 
rates compression test analysis, this article analyze the properties of the underwater blast wave protection materials used on life jacket, and the effects of foam density and yield strength to the energy absorption performance. The experimental results are valuable to underwater blast wave protection materials research and develop.

\section{Experimental}

Materials. Considering used on life jackets, the shock wave protection materials can't whittle down the flotation capability of the life jackets. So the densities of the protection materials should as small as possible, and should have better flexibility for wearing comfort. According to the density of the active military life jacket flotation body NBR/PVC foam, we trial produce four kinds of low densities closed cell polyurethane foams which the densities verge on the NBR/PVC foam. The microstructure picture of one trial product sample and NBR/PVC foam is show in Fig.1. Table 1 lists the primary parameters of the five test materials.

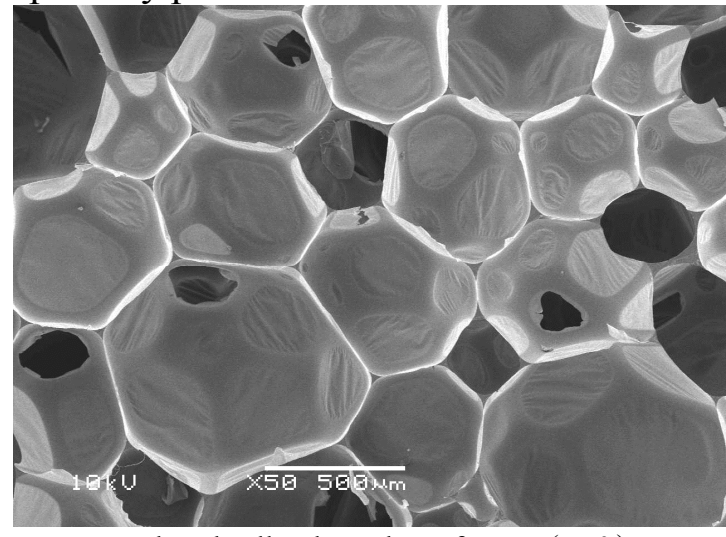

closed cell polyurethane foam $(\times 50)$

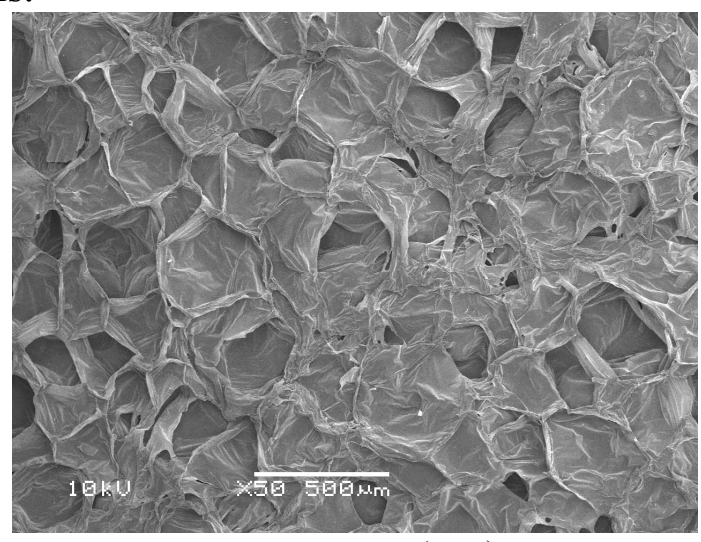

NBR/PVC foam $(\times 50)$

Fig.1 SEM images of closed cell polyurethane foam and NBR/PVC foam

Table 1 The testing materials samples parameters

\begin{tabular}{cccc}
\hline Sample No. & Average Cell Size $(\mu \mathrm{m})$ & Density $\left(\mathrm{g} / \mathrm{cm}^{3}\right)$ & Porosity \\
\hline Foam 1 & 832.5 & 0.024 & $90.2 \%$ \\
\hline Foam 2 & 529.1 & 0.029 & $87.2 \%$ \\
\hline Foam 3 & 673.7 & 0.038 & $92.5 \%$ \\
\hline Foam 4 & 759.1 & 0.045 & $91.1 \%$ \\
\hline NBR/PVC & 257.3 & 0.034 & $61.6 \%$ \\
\hline
\end{tabular}

Method. Quasi-static compression tests were carried out by the MTS Bionix Servohydrolic Test System, Eden Prairie, MN, USA.(Fig.2). The foams were cut into $50 \times 50 \times 20 \mathrm{~mm}$ cubes. Axial compression rates were $1.2 \mathrm{~mm} / \mathrm{min}, 12 \mathrm{~mm} / \mathrm{min}$ and $120 \mathrm{~mm} / \mathrm{min}$, and the strain rates range of $10^{-3}$ $\sim 10^{-1} \mathrm{~s}^{-1}$ were acquired. The sample under quasi-static compression before and after is shown in Fig.3.
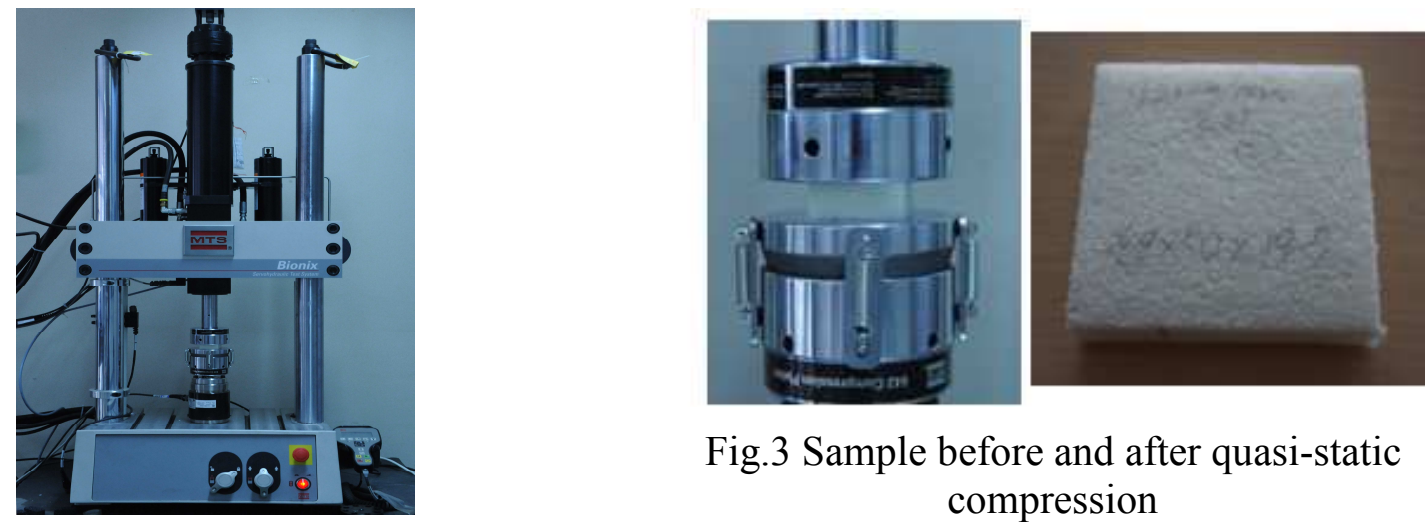

Fig.3 Sample before and after quasi-static compression

Fig.2 Quasi-static compression tester 
Dynamic compression tests were taken with Split Hopkinson Bar (Fig.4). Because the wave impedance of the foams is very low, the transmission signal will be very weak if using traditional SHPB. The pressure bar is made in magnesium alloy, and the density is $1.9 \mathrm{~g} / \mathrm{cm}^{3}$, and Young modulus is 43GPa. The signal acquisition use advanced Multichannel Genesis Transient Waveform Recorder, which can meet the accuracy requirements. To reflect the true properties, the sample size is at least ten times of the cell size. Considering the influence of above mentioned factors and the pressure bar diameter to wave transmission, the pressure bar diameter is $30 \mathrm{~mm}$, and sample diameter is $25 \mathrm{~mm}$, height $10 \mathrm{~mm}$. To satisfy the condition of samples stress state uniformity much better, the bullet length is $600 \mathrm{~mm}$. The sample under dynamic compression before and after is shown in Fig.5.

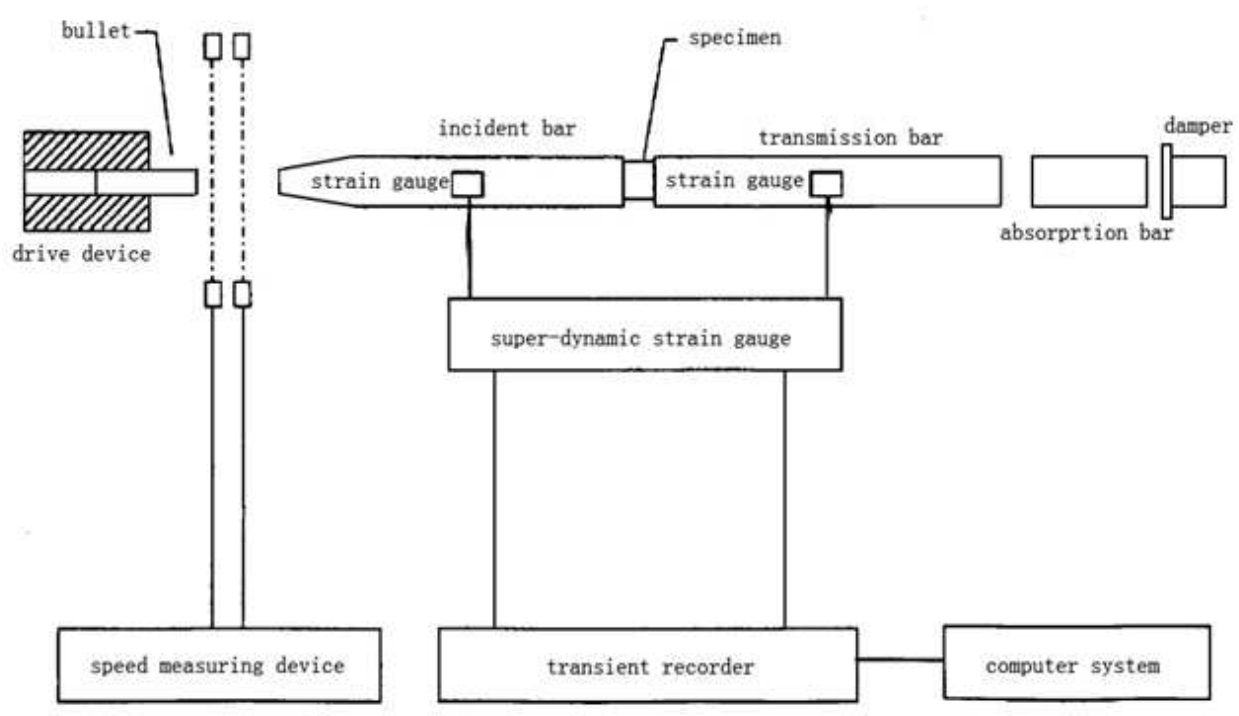

Fig.4 Sketch of Split Hopkinson Pressure Bar
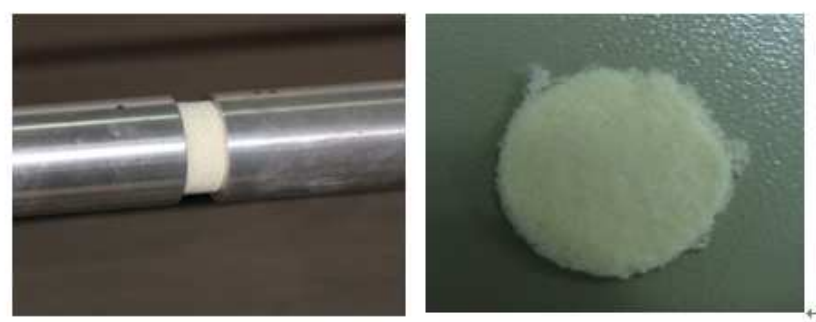

Fig.5 Sample before and after dynamic compression

The experimental technology of SHPB is based on two basic suppositions: one-dimensional supposition and uniform supposition. The strain rate, strain and stress of the test materials are calculated with the theory of one-dimensional stress wave transmission. The equation of strain rate, strain and stress is expressed as follows.

$$
\begin{aligned}
& \dot{\varepsilon}(t)=\frac{C_{0}}{L}\left[\varepsilon_{I}(t)-\varepsilon_{R}(t)-\varepsilon_{T}(t)\right] \\
& \varepsilon(t)=\frac{C_{0}}{L} \int_{0}^{t}\left[\varepsilon_{I}(t)-\varepsilon_{R}(t)-\varepsilon_{T}(t)\right] d t \\
& \sigma(t)=\frac{A E}{2 A_{0}}\left[\varepsilon_{I}(t)+\varepsilon_{R}(t)+\varepsilon_{T}(t)\right]
\end{aligned}
$$




\section{Results and Discussion}

Five Testing Materials Energy Absorbing Properties Comparison . Figure 6 shows stress-strain curves of the four low density closed cell polyurethane foams under quasi-static compression.

As shown in Fig.6, the deformation of four low densities closed cell polyurethane foams can be marked off three phase under quasi-static compression load: elasticity zone, yield plateau zone and densification zone. At the yield plateau zone, the strain rates reach $65 \%$ and even more, but the plateau stress holding the line. The stress-strain curves of the NBR/PVC foam is shown in Fig.7. In Fig. 6 and Fig.7, the five testing materials are all show distinctive strain rates reinforcement effects. Along with the strain rates increase, the elastic yield plateau strength of the materials show elevation trends, but the extensibility changes are not obvious. With the increase of density, the yield strength increase also. It is indicated that the influence of the densities to yield strength is distinctive.

Ideal protection layer material should have a wide yield plateau zone and come into yield plateau zone rapidly after a minor elastic deformation, and then come into the compaction phase when under compression. In the way of force transmission, the maximum pressure of porous materials transmission is its yield pressure before compaction. The force excess yield pressure is absorbed by the material plastic deformation. That is the protection mechanism of porous material. Accordingly, the wider of the protection layer material yield plateau and the bigger of the dimensions under stress strain curves, the better of the materials energy absorbing capacity. We adopt two criterions to contrast the samples blast wave protection properties. One is the foam yield plateau strength, and the other is the foam energy absorbing capacity. According to the domestic and foreign achievements on underwater blast injury ${ }^{[5-9]}$, considering the hazard of water shock wave to the ship's complement in water during war, we consider $0.4 \mathrm{MPa}$ as the protection materials yield plateau strength threshold. Because the underwater blast wave peak pressure smaller than the impact strength can't bring fatal-wound, and light underwater blast injuries which the impact strength may cause will recruitment after reach medical aid timely. So at the phase of foam plastic deformation, the plateau yield stress can't excess $0.4 \mathrm{MPa}$, the protection is meaningless or else. The primary energy absorbing phase of the foam is in yield plateau zone, so we compare the energy absorbing capacity by the plastic plateau power density, namely the amount of energy absorbing in plastic plateau zone.
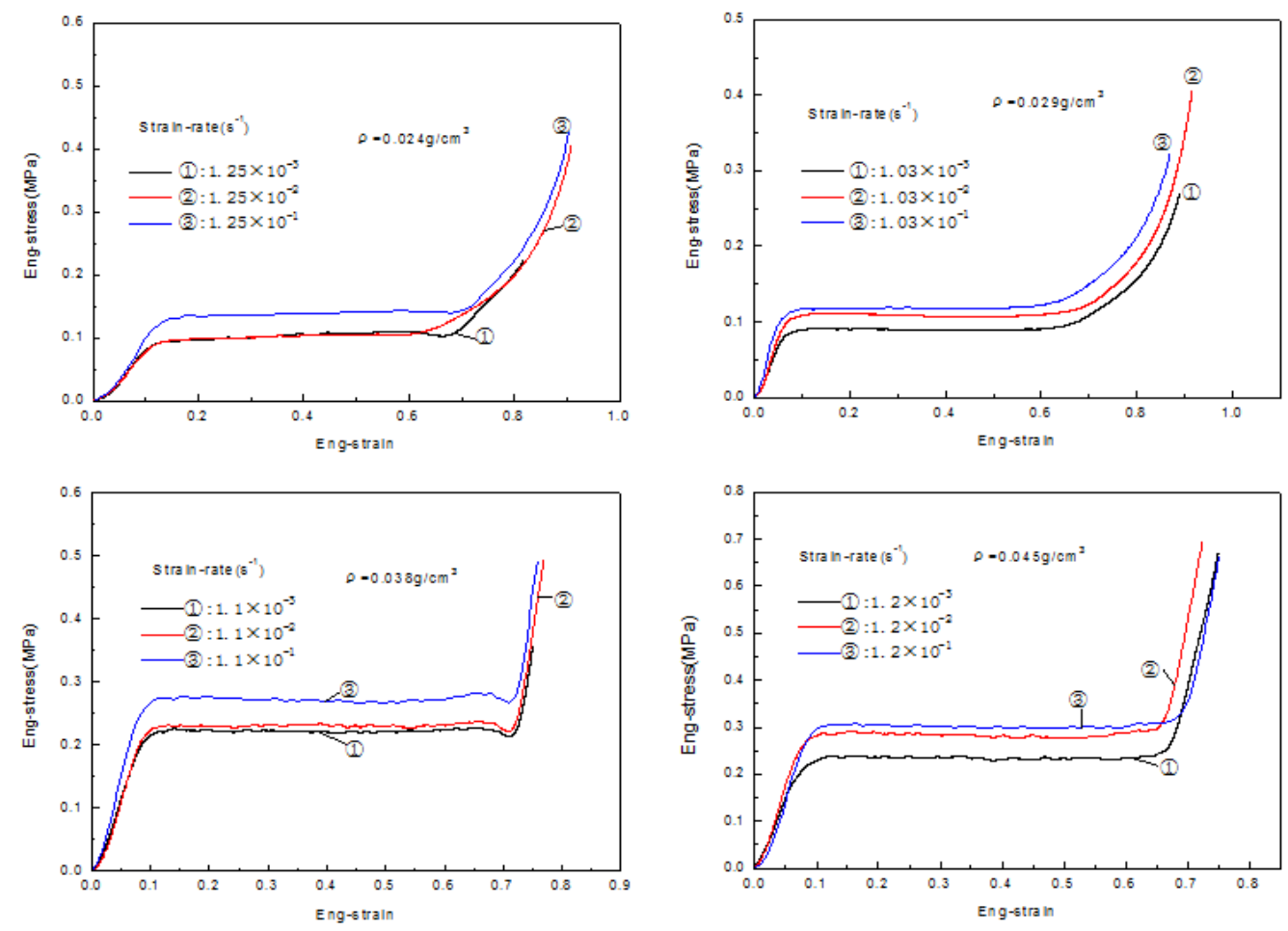

Fig.6 Quasi-static compression stress-strain curves of polyurethane foams 

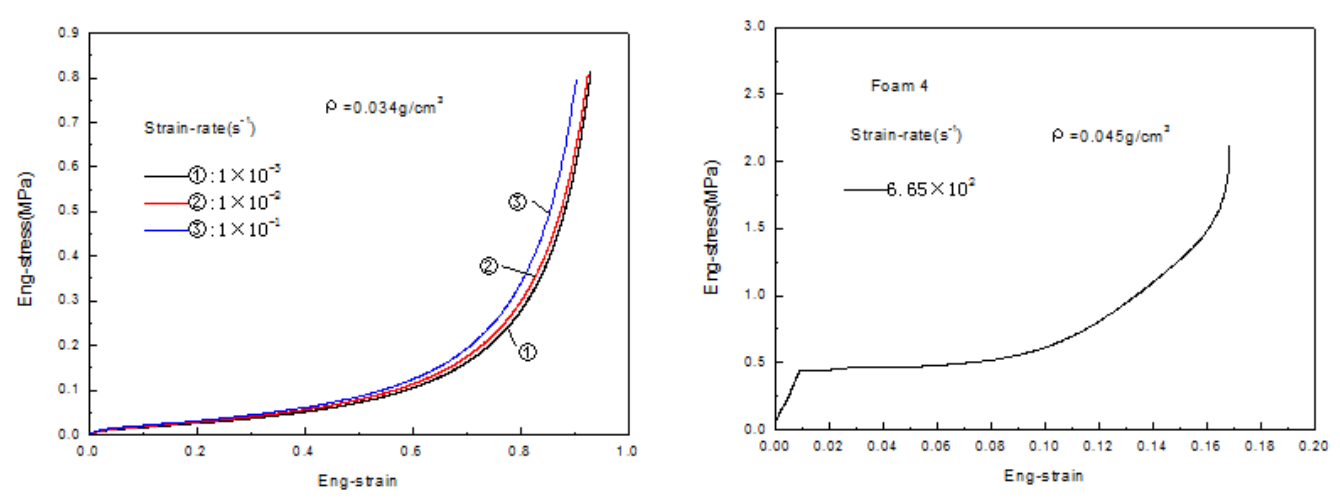

Fig.7 Quasi-static compression stress-strain curves of NBR/PVC foam

Fig. 8 Dynamic compression stress-strain curve of one trial polyurethane foam

Table 2 The testing samples plastic plateau power density at low strain rates

\begin{tabular}{lccc}
\hline \multirow{2}{*}{ Sample No. } & \multicolumn{3}{c}{ Power Density $\left(\mathrm{J} / \mathrm{m}^{3}\right)$} \\
\cline { 2 - 4 } & $10^{-3} \mathrm{~s}^{-1}$ & $\begin{array}{c}\text { Strain Rates } \\
10^{-2} \mathrm{~s}^{-1}\end{array}$ & $10^{-1} \mathrm{~s}^{-1}$ \\
\hline Foam 1 & $5.5 \times 10^{4}$ & $6.7 \times 10^{4}$ & $8.5 \times 10^{4}$ \\
\hline Foam 2 & $6.5 \times 10^{4}$ & $7.7 \times 10^{4}$ & $8.2 \times 10^{4}$ \\
\hline Foam 3 & $1.36 \times 10^{5}$ & $1.39 \times 10^{5}$ & $1.55 \times 10^{5}$ \\
\hline Foam 4 & $1.30 \times 10^{5}$ & $1.51 \times 10^{5}$ & $1.68 \times 10^{5}$ \\
\hline NBR/PVC & $3.7 \times 10^{4}$ & $3.9 \times 10^{4}$ & $4.5 \times 10^{4}$ \\
\hline
\end{tabular}

From table 2, we can find that with the increase of strain rates and density, the plastic plateau power density of the foams increase too.

The results also show that the polyurethane foams are a good energy absorbing materials. The plastic plateau power density of the NBR/PVC foams is smaller than other foams.

Dynamic yield Stress Contrast. It has been stated above that polyurethane foam shows distinctive strain rates reinforcement effect, and the plastic yield plateau strength shows elevation trends along with the strain rates increase. Generally, the porous materials strain rates are at 102s-1 around under the water blast wave impact. To guarantee the yield plateau stress can't excess the threshold pressure of light underwater blast injury, the dynamic yield strengths of four polyurethane foams have been tested with SHPB. The typical stress-strain curve is shown in Fig.8.

Table 3 The yield strength of four polyurethane foams

\begin{tabular}{ccccc}
\hline & \multicolumn{4}{c}{ Yield strength (Pa) } \\
\cline { 2 - 5 } Sample No. & \multicolumn{3}{c}{ Strain Rates } \\
& $1 \times 10^{-3} \mathrm{~s}^{-1}$ & $1 \times 10^{-2} \mathrm{~s}^{-1}$ & $1 \times 10^{-1} \mathrm{~s}^{-1}$ & $6.7 \times 10^{2} \mathrm{~s}^{-1}$ \\
\hline Foam 1 & $9 \times 10^{4}$ & $9 \times 10^{4}$ & $1.4 \times 10^{5}$ & $5.6 \times 10^{5}$ \\
\hline Foam 2 & $9 \times 10^{4}$ & $1.1 \times 10^{5}$ & $1.2 \times 10^{5}$ & $3.9 \times 10^{5}$ \\
\hline Foam 3 & $2.2 \times 10^{5}$ & $2.3 \times 10^{5}$ & $2.6 \times 10^{5}$ & $4.6 \times 10^{5}$ \\
\hline Foam 4 & $2.3 \times 10^{5}$ & $2.8 \times 10^{5}$ & $3 \times 10^{5}$ & $4.7 \times 10^{5}$ \\
\hline
\end{tabular}

Table 3 lists the yield strength of four testing polyurethane foams from low strain rates to higher strain rates. It is shown that the dynamic yield strength of foam 2 is close to $0.4 \mathrm{MPa}$ at higher strain rate. 


\section{Conclusions}

This paper experimentally explores the energy absorbing properties of underwater blast wave protection materials used on life jacket. It is seen from the test results that the low density closed cell polyurethane foams are sensitive to strain rates. The dynamic yield plateau strength and plastic plateau power density can be considered as two criterions for underwater blast wave protection materials select. The protection material research and application can prevent and alleviate the underwater blast injury happen dramatically in sea warfare and underwater engineering blast. Combined with the test results and discussion, it can be concluded that the polyurethane foam can be used as underwater blast wave protection layer materials. To obtain better protection effect, detailed work on the relationship between the protection material and the floating body of life jacket under compression load needs to be done in future.

\section{References}

[1] Wakely, C. P. G. Effect of Underwater Explosions on the Human Body. The Lancet pp 715-718 (1945)

[2] White cs. Biological blast effects. TID-5564, 1959.

[3] Wolf NM. Underwater Blast Injury-A Review of the Literature. US Naval Submarine Medical Center, Report No. 646,1970

[4] Richmond DR, etc. A tentative estimation of man's tolerance to overpressures from air blast. AD $293875,1962$.

[5] WANG Zhong-qian. On the safety distance for the protection of the human body during the operation of underwater blast. Journal of Blast and Shock. 1982(1):109-116

[6] Hartman, G. K. The physical aspects of underwater explosion damage to swimmers. National Research Council Report NRC CAO: 0034 (1953)

[7] WANG Zheng-guo. Blast injury. 1st ed. Beijing: People's Military Surgeon Press, 1983:82.

[8] WANG Zheng-guo. Blast injury research. the 4th Shock Tube and Shock Wave Academic Convention [C]. Nanjing: 1984:12

[9] John A. Lewis. Effects of underwater explosions on life in the sea. DSTO Aeronautical and Maritime Research Laboratory, 1996.8:24 


\section{Advanced Materials Research II}

10.4028/www.scientific.net/AMR.463-464

Experimental Study of the Compressive Performance of Life Jacket Use Polyurethane Foam for Blast Wave Protection

10.4028/www.scientific.net/AMR.463-464.457 